\title{
Peripheral arterial pseudoaneurysm treated by ultrasound-guided compression
}

\author{
Yun-Chung Cheung, Yung-Liang Wan
}

\begin{abstract}
Summary
Ultrasound-guided compression repair of a pseudoaneurysm is a simple, safe and effective therapy, and has been proposed as a first-line treatment for pseudoaneurysms caused by catheterization. Herein, we report a case of a pseudoaneurysm arising from a sport injury which was treated by this method. Obliteration of the track of the pseudoaneurysm induced manual thrombosis and successfully converted the pseudoaneurysm to haematoma. Understanding the mechanism and the procedure is the basis of success in the use of this treatment.
\end{abstract}

Keywords: ultrasound, pseudoaneurysm

Surgical repair of peripheral arterial pseudoaneurysm is safe and effective, but it may prolong the duration of hospitalisation and be complicated by wound infections, pain and scarring. Recently, ultrasound-guided compression repair (UGCR) of a pseudoaneurysm has been accepted as an alternative to surgical management. ${ }^{1-5}$ In a review of the radiological literature, all the reported cases are pseudoaneurysms caused by catheterisation of either femoral or brachial arteries. We report a case of pseudoaneurysm after sport injury which was treated by UGCR.

\section{Case report}

A healthy 17-year-old boy sprained his right ankle during a basketball match. He visited a Chinese herb doctor for massage therapy. After

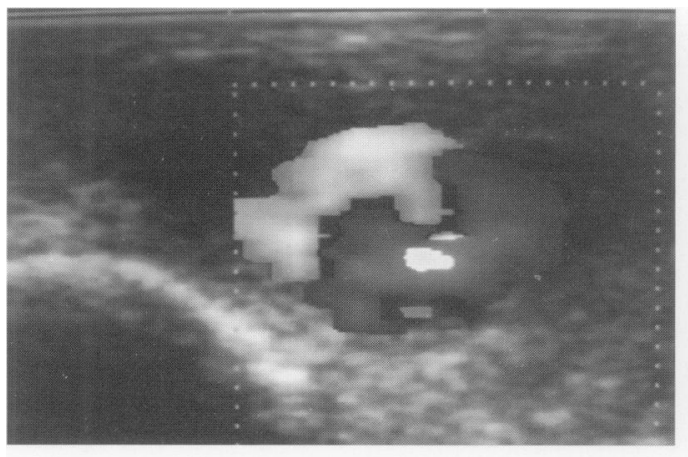

A

Figure 1 (A) Transverse sonogram of the aneurysm shows typical swirling pattern of colour flow within the mass; (B) Longitudinal sonogram of the mass shows a short and narrow track, between the pseudoaneurysm and the artery (arrows) the massage, he felt much better, but a progressively enlarging mass was discovered over his right ankle in the next morning. He presented to our emergency department with an egg-sized pulsatile mass over the lateral aspect of his right ankle. Under the tentative diagnosis of haematoma, a needle aspiration was done. An automatic infusion into the syringe with fresh blood was seen. Highresolution ultrasonography with $7.5 \mathrm{MHz}$ transducer showed an anechoic mass measured $0.8 \mathrm{~cm} \times 1 \mathrm{~cm}$ with a swirling pattern of internal colour flow (figure 1A) and a short, narrow track (figure 1B). Pseudoaneurysm was diagnosed.

Under ultrasonographic guidance, compression by the $7.5 \mathrm{MHz}$ transducer was gradually applied until the colour flow within the pseudoaneurysm disappeared. The compression was maintained for 15 minutes. Compression cycles were continued until the cessation of abnormal flow was apparent after the force was released. After two sessions of compression, the cystic mass was replaced by an echogenic haematoma (figure 2). No colour flow was identified in follow-up scans obtained after 1,2, and 5 minutes and after 24 hours. The ankle was then carefully banded and fixed for one week. There was no evidence of recurrent pseudoaneurysm or any complications.

\section{Discussion}

UGCR as an alternative to surgery has recently been proposed for postcatheterisation arterial injuries complicated by pseudoaneurysms and arteriovenous fistulas. The method is simple

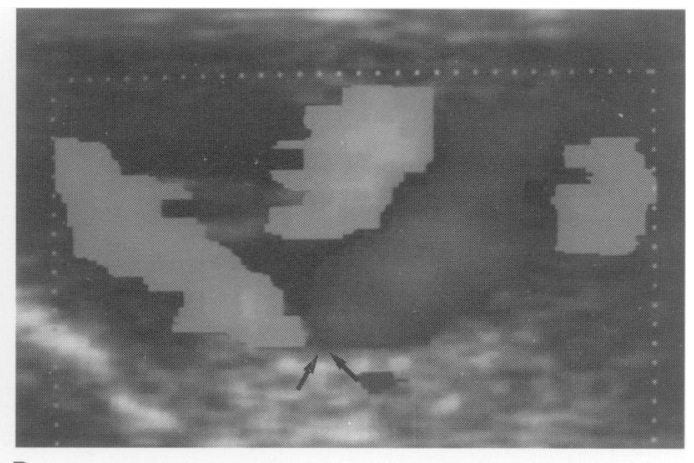

B 


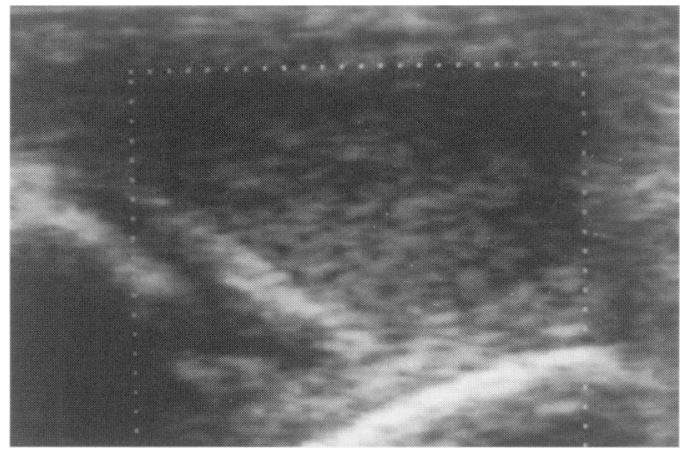

Figure 2 Sonogram of the mass five minutes after UGCR demonstrates an echogenic mass replacing the original pseudoaneurysm

and effective with a reported success rate ranging from $71 \%$ to $93 \% .^{1-5}$ Some small pseudoaneurysms are able to thrombose spontaneously, ${ }^{7}$ but many do not; a safe, effective, treatment such as UGCR may eliminate the need for prolonged observation and delayed treatment and reduce the risk of spontaneous rupture of a pseudoaneurysm.

The pseudoaneurysm in this report was not caused by catheterisation, but probably by either the sprain during sport or by massage. The mechanism of and rationale for using UGCR to resolve pseudoaneurysms from different aetiologies are the same, however. ${ }^{1-7}$ Obliteration of the track of the pseudoaneurysm and cessation of the blood flow will induce thrombosis, and facilitate the formation of a haemostatic plug and sequential conversion to a simple haematoma, which will ultimately resolve spontaneously. Thus, it seems reasonable that the success rate relates closely to the thrombogenicity and morphology of the track. The chronicity of a pseudoaneurysm will

1 Fellmeth BD, Roberts AC, Bookstein J, et al. Postangiographyc femoral artery injuries: nonsurgical repair with USguided compression. Radiology 1991; 178: 671-5.

2 Fellmeth BD, Buckner NK, Ferreira JA, Rooker KT, Parsons PM, Brown PR. Postcatheterization femoral artery injuries: repair with color flow US guidance and C-clamp assistance. Radiology 1992; 182: 570-2.

3 Fellmeth BD, Baron SB, Brown PR, et al. Repair of postcatheterization femoral pseudoaneurysms by color flow ultrasound guided compression. Am Heart 7 1992; 123: 547-51.

4 Schwend RB, Hambsch KP, Kwan KY, Boyajian RA, Otis SM. Color duplex sonographically guided obliteration of pseudoanerysm. F Ultrasound Med 1993; 12: 609-13. decrease the thrombogenicity of the track due to the development of endothelium. Longstanding pseudoaneurysm, more than onemonth old, has previously been regarded as untreatable, while a long and narrow track is easier to close than a short, broad one. ${ }^{1}$ Leg ischaemia and pseudoaneurysmal rupture are the potential morbidities, even though they are rarely reported.

As UGCR is a time-consuming procedure, operator fatigue is a critical factor, while some authors consider that a compression device may increase the success rate appreciably. ${ }^{2,8}$ The superficial nature of the pseudoaneurysm in our case enabled us to maintain compression without any device. The acuity of the pseudoaneurysm and the tolerance of the patient also contributed to our success.

Traditionally, a surgical approach is considered first when a pseudoaneurysm is discovered. We believe that, in suitable cases, an attempt should first be made using UGCR, since the technique is safe and cost-effective and surgery can always be employed if UGCR fails. Not all cases are suitable for UGCR, however. Severe tenderness may preclude compression, the presence of infection, a very large or hard haematoma, and critical leg ischaemia require surgical intervention rather than UGCR.

In conclusion, UGCR is a simple and costeffective first-line therapy, not only for postcatheterisation pseudoaneurysm, but also for peripheral arterial pseudoaneurysm after sport injury. In our case, there was a significant decrease of blood flow within the pseudoaneurysm after the first 15 minutes of compression. The location and acuity of the pseudoaneurysm and the cooperation of the patient are also important for the success of UGCR.

5 Tschammler A, Elsner H, Jenett $M$, et al. Compression repair of femoral postcatheterization pseudoaneurysm guided by color doppler flow imaging. Eur Radiol 1995; 5: $285-7$

6 Skibo L, Polak JF. Compression repair of a postcatheterization pseudoaneurysm of the brachial artery under sonographic guidance. $A f R$ 1993; 160: 383-4.

7 Kotval PS, Khoury A, Shah PM, Babu SC. Doppler sonographic demonstration of the progressive spontaneous thrombosis of pseudoaneurysms. F Ultrasound Med 1990; 9 . 185-90.

8 Trerotola SO, Savader SJ, Prescott CA, Osterman FA. USguided pseudoaneurysm repair with a compression device. Radiology 1993; 189: 285-6. 